\title{
Morphine and Dexamethasone Incorporated
}

Cocktail Regimen Efficiently Reduced Postoperative Pain in Patients Undergoing Primary Total Knee Arthroplasty

\author{
Jian Gong \\ Dalian medical University \\ Yuancheng Li \\ Dalian Medical University \\ Shi Liu \\ Dalian Medical University \\ Cui XU \\ Dalian Medical University \\ Ying Gong ( $\square$ gying202110@163.com ) \\ The First Affiliated Hospital of Dalian Medical University
}

\section{Research article}

Keywords: Morphine, Dexamethasone, Total knee arthroplasty, Cocktail Regimen, Postoperative Pain

Posted Date: November 1st, 2021

DOI: https://doi.org/10.21203/rs.3.rs-983406/v1

License: (a) (i) This work is licensed under a Creative Commons Attribution 4.0 International License.

Read Full License 


\section{Abstract}

Purpose: The present study was conducted to evaluate the pain-reducing efficacy and safety of the morphine and dexamethasone incorporated cocktail regimen in patients undergoing total hip arthroplasty (THA).

Methods: The current study enrolled 75 patients and randomized them into three groups. The patients in group I were injected with morphine, dexamethasone, bupivacaine, flurbiprofen axetil, and normal saline. Patients assigned to group II were injected with dexamethasone, bupivacaine, flurbiprofen axetil, and normal saline. Patients in group III were given bupivacaine, flurbiprofen axetil, and normal saline. The visual analog scale (VAS) score and active and passive range of movement (ROM) were recorded and evaluated to compare the pain-controlling efficiency. The side effects of headache, dizziness, nausea, vomiting, wound leakage, and wound infection were monitored.

Results: The patients in group I had the lowest VAS, and highest active ROM and passive ROM. The VAS reduced from day 1 to day 4, and the ROM increased conversely. The patients in group II presented a smaller reduction in VAS and a smaller increase in ROM, compared to the patients in Group III. The side effects of headache, dizziness, nausea, and vomiting were not recorded. Wound leakage was observed from 4 patients in group I ( 2 patients) and group II ( 2 patients). The was no statistical intergroup difference $(p=0.85)$. No wound infection symptoms were detected.

Conclusions: Morphine and dexamethasone incorporated cocktail regimen efficiently reduced postoperative pain in patients undergoing primary total knee arthroplasty.

Trial registration: Drug clinical trial registration system, CTR20181569. Registered 20 October 2018, http://www.chinadrugtrials.org.cn/clinicaltrials

\section{Introduction}

Total knee arthroplasty (TKA) has been regarded as one of the most successful surgeries with satisfying outcomes including evident restored joint movement and significantly reduced pain in the long run.[1] However, patients undergoing TKA usually suffer severe postoperative pain, which slowed the postoperative recovery and increased the hospital stay and cost.[2], Without adequate pain management, severe postoperative pain causes heavy stress on the cardiovascular system, which could potentially result in serious complications, such as ischemic cardiac events and myocardial insufficiency.[3] In addition, immobilization caused by pain increased the risk of complications, such as ileus and thrombus formation.[4] The severe pain could lead to hormone imbalance and sleep disorder, which can cause immune system dysfunction and a higher risk of infection.[5] In the end, uncontrolled pain could cause delirium or anxiety disorder, especially in elder patients.[6]

Local Infiltration Analgesia (LIA) is a simple, practical, safe, and effective strategy for pain controlling after knee and hip surgery.[7] Intraoperative periarticular injection of the multimodal cocktail has been 
widely used for ages to reduce pain and its analgesic effect has been considered comparable to the nerve block technique.[8] Nowadays, cocktail injection is popularly injected into muscles, tendons, and bursa around the knee joint.[8] The injection mixture was consisted of multiple compositions, including opioids, anesthetics, corticosteroids, and non-steroidal anti-inflammatory drugs (NSAIDs).[9] However, there was still no gold standard regimen of the cocktail ingredient, and the proper dosage and composition of the injection cocktail have not reached an agreement. It's mainly because of the potential complications brought by using opioids and corticosteroids. Opioids act on m-receptors that are distributed widely in the cerebral cortex, thalamus, medulla oblongata, spinal cord, and primary sensory neurons in the central nervous system.[10] Accordingly, opioids usually demonstrated a strong analgesic effect. On the other side, the overuse of opioids usually causes side events, including headache, dizziness, nausea, vomiting, which limited the recovery speed after surgery.[11] Over the past decades, corticosteroids (CS) have emerged as steroidal medicine to reduce systematic inflammation caused by rheumatoid arthritis, systemic lupus erythematosus, and asthma. Corticosteroids have been widely used for reduced inflammation by reducing the release of various inflammatory factors, inhibit white blood cell infiltration and phagocytosis, thereby reducing pain and eliminating red.[12] Meanwhile, by increasing vascular tension and reducing the capillary permeability, and thus reduce exudation and edema.[13] But it should be noted that inflammation is a defensive response of the body, and the reaction of inflammation is an important process of tissue repair. Therefore, improper use of this class of drugs can cause the spread of infection and delayed wound healing.

In the current study, we have designed three different periarticular cocktail regimens and compared their postoperative pain-reducing efficiency for patients with TKA. The candidate ingredients are morphine (5 $\mathrm{mg}$ ) and/or dexamethasone (5 mg), and the basic components were bupivacaine (10 mg) and flurbiprofen axetil $(10 \mathrm{mg}$ ). The visual analog scale (VAS) score and range of movement (ROM) were recorded and evaluated to compare the pain-controlling efficiency. Simultaneously, the potential converse effects accompanied with the application of morphine and dexamethasone were also monitored to assess the tolerability of the regimens.

\section{Materials And Methods}

\subsection{Patients enrollment}

From September 2018 to October 2020, a total of 90 patients aged between 50-70 scheduled for elective primary TKA were assessed for the eligibility $f(F i g .1)$. All the patients diagnosed with knee osteoarthritis were included in this study. Patients were excluded when they were diagnosed with rheumatoid arthritis (RA), or patients who have a history of knee replacement surgery, hepatic dysfunction, or renal dysfunction, or ischemic heart disease. In the end, 75 patients were enrolled, and 15 patients were excluded. The enrolled patients were randomized into 3 groups. The demographic statistics, preoperative pain evaluation data, and ROM assessment were recorded and presented in Figure 1.

\subsection{Administration of Intraoperative Periarticular Injection}


The regimens of the cocktails were presented in Table 2. The patients in group I were injected with morphine (5 mg), dexamethasone (5 mg), bupivacaine (10 mg), flurbiprofen axetil (10 mg), and normal saline (20) $\mathrm{mL}$. For patients assigned to group II, dexamethasone $(5 \mathrm{mg})$, bupivacaine (10 mg), flurbiprofen axetil(10 mg), and normal saline $(20 \mathrm{~mL})$ was injected into the joint space. Patients in group III were given bupivacaine $(10 \mathrm{mg})$, flurbiprofen axetil冈10 $\mathrm{mg})$, and normal saline $(20 \mathrm{~mL})$.

\subsection{Surgical Procedure and Perioperative Management}

All surgical procedures were conducted in our hospital by the same surgical team using the same approach. Total Knee replacement systems were provided by (GENESIS II; Smith \& Nephew, London, United Kingdom). All procedures were performed by the same knee medial parapatellar approach, under general anesthesia. Cocktails were injected at the fixed locations illustrated in Figure 2 after the osteotomy was finished.

\subsection{Outcome Assessment}

The outcomes mainly include dynamic observation VAS score, active ROM, and passive ROM in the first four days after TKA surgery. Potential side effects including headache, dizziness, nausea, vomiting were monitored. During the hospital stay, complications (wound leakage and superficial infection) and other adverse events (cardiac infarction, stroke, and acute renal failure) were also carefully monitored.

\subsection{Statistical Analysis}

The SPSS software (version 19.0; IBM, Chicago, IL) was applied to analyze statistical differences with the one-way ANOVA and Tukey 's Post Hoc Test to determine the significances of group differences in continuous variables. The Pearson Chi-squared test or Fisher exact test was applied to analyses qualitative comparative parameters. A significant difference was defined when $\mathrm{P}<0.05$.

\section{Results}

The study enrolled 90 patients who were performed with TKA in our hospital from September 2018 to October 2020. According to the exclusion criteria, 15 patients were excluded based on the exclusion criteria. The remaining 75 patients were included with 25 patients randomized to Group I, 25 to Group II, and 25 to Group III (Figure 1).

The general demographic characteristics including age, gender, body mass index (BMI), and preoperative data were compared between the 3 groups (Table 1). After TKA, The VAS scores, active ROM, and passive ROM of all the groups were presented in Table 3, Table 4, and Table 5, respectively.

As shown in Table 3, patients in group I had the lowest VAS, and highest active ROM and passive ROM. The VAS reduced from day 1 to day 4, and the ROM increased conversely (Table 4 and Table 5). The patients in group II presented a smaller reduction in VAS and a smaller increase in ROM, compared to the patients in Group III. 
The side effects of headache, dizziness, nausea, and vomiting were not recorded. Wound leakage was observed from 4 patients in group I ( 2 patients) and group II (2 patients). The intergroup difference was not observed $(p=0.85)$. No wound infection symptoms were detected. All the enrolled patients presented similar serum C-reactive protein and erythrocyte sedimentation rate $(\mathrm{mm} / \mathrm{h})$ levels (Table 6$)$. Diverse events like cardiac infarction, stroke, or acute renal failure had not been recorded. Patients in all three groups presented similar hospital stays and costs.

\section{Discussion}

Total knee arthroplasty (TKA) is one of the most common surgeries performed nowadays. However, severe postoperative pain after TKA made the patient suffering and limit postoperative recovery. The periarticular cocktail injection was widely used in TKA to effectively reduce pain[14, 15], although there is no consensus yet of the ingredients. The current study was designed to compare three cocktail regimens for postoperative analgesia and assess the potential side effects of added morphine and dexamethasone.

The patients in Group I demonstrated the lowest VAS scores in the first 4 days after TKA, which confirmed the regimen effectively reduce the damaging stimulus and the release of inflammatory mediators, prevent pain sensitization and alienation, and achieve balanced analgesia. It was due to the main components in the regimen work synergistically on different targets. Bupivacaine is a long-acting amide local anesthetic. It mainly inhibits the sodium ion channel of the nerve cell membrane to block nerve excitement and conduction.[16] Its anesthesia intensity is 16 times that of procaine and 2-3 times that of lidocaine, and the duration of action is 8 times that of procaine. $[16,17]$ The surface of periosteum was covered by a layer of dense fibrous membrane. The internal surface of the periosteum is rich in unmyelinated nerve fibers and small vessels. The small unmyelinated nerve fibers in the periosteum are sensitive to local anesthetic. Thus, the regimen could provide a basic pain-controlled effect due to bupivacaine.

Flurbiprofen axetil is a non-steroidal analgesic with lipid microspheres as the drug carrier.[18, 19] After the drug was injected into the body, flurbiprofen axetil is released from the lipid microspheres, and is rapidly hydrolyzed under the action of carboxylesterase to produce flurbiprofen[20], which inhibits the synthesis of prostaglandins and reduce pain.[21, 22] Morphine is a complete agonist of opioid receptors. It can mimic endogenous opioid active substances by binding to opioid receptors in different brain regions to exert pharmacological effects, such as with dorsal thalamus, ventricles, peri-aqueduct gray matter, and spinal cord glue.[23, 24] Compared to intermittent sharp pain and visceral colic, morphine has a better effect on persistent dull pain than[25], which is ideal for reducing the postoperative pain after TKA. Opioids usually exert an analgesic effect 10-20 minutes after one dose, the strongest effect is 1 to 2 hours, and it can be maintained for 4-6 hours. [26] Morphine also has a significant sedative effect, which is related to the binding of opioid receptors in the limbic system to eliminate emotional changes caused by pain and can eliminate anxiety caused by pain.[27] Emotional reactions such as tension and fear, thus significantly improving the tolerance to pain. $[28,29]$ Steroids have anti-inflammatory effects and may reduce these effects by inhibiting the cyclooxygenase pathway, stabilizing neuronal cell membranes, and reducing the level of bradykinin in the tissue.[30] Dexamethasone has also been shown to inhibit the 
release of neuropeptides from nerve endings after tissue injury, such as calcitonin gene-related peptide and substance $P$, which were both for reducing pain.[31, 32]

The patients in Group I also demonstrated the biggest active and passive VOM in the first 4 days. It was partially due to the pain was reduced to the lowest level, so the range of passive knee movement could achieve the highest level. The reduced pain also encouraged the patients to conduct the active exercise. The good ROM could also be partially reasoned with the use of corticosteroids. More than directly suppressing inflammation, corticosteroids also increase vasoconstriction, reduce vascular permeability, antagonize the expansion of blood vessels by inflammatory mediators such as histamine, reduce local congestion, and inhibit the exudation of white blood cells and body fluids. Thus, corticosteroids used local efficiently reduce exudation and edema, which was beneficial for achieving a higher ROM in the beginning.

No side effects were observed during the hospital stay, it was mainly because the morphine and dexamethasone were used in a small amount. In addition, the applied dexamethasone decreased capillary permeability, so the morphine entered the blood circulation at a slow rate, so the risk of side effects related to morphine was minimized. The well-controlled pain and limb swelling are beneficial for early rehabilitation after TKA, which could help to restore the general condition and immune system. Therefore, the infection was not observed by monitoring serum CRP and ESR continuously.

This current study possessed several limitations though. First, the conducted study was a single-center study, and the sample size was relatively small. Future studies with a larger sample size conducted in multicenter might potentially help find a clearer intergroup difference. Second, although all the surgical processes were conducted by the same surgical team, the different requirements of osteotomy and soft tissue release of specific individuals might have a certain but undefined influence on pain and movement evaluation. Finally, in the present study, the outcomes were analyzed largely based on VAS evaluation, which was a subjective rating scale. As different patients possess a different sensitivity and accuracy of expression to pain, so the VAS was inevitably biased. An objective rating scale was needed in the future to precisely record and compare the outcome.

In conclusion, for patients undergoing primary unilateral TKA, the morphine and dexamethasone incorporated cocktail regimen significantly reduced postoperative pain and promoted ROM which was beneficial for early postoperative enhanced recovery. The combined cocktail did not raise any side effects.

\section{Declarations}

\section{Ethics approval and consent to participate}

All procedures performed in this study were following the ethical standards of the Shanxi Great hospital research committee and with the 1964 Helsinki declaration and its later amendments or comparable ethical standards. 
All patients enrolled in this study had signed a consent form to participate this the trial.

\section{Consent for publication}

Not applicable.

\section{Availability of data and materials}

The datasets during and/or analyzed during the current study are available from the corresponding author on reasonable request.

All data generated or analyzed during this study are included in this published article [and its supplementary information files].

\section{Competing interests}

The authors declare there is no conflict of interest.

\section{Funding}

This study was not funded.

\section{Authors' contributions}

Jian Gong collected the data and wrote the manuscript, Yuancheng Li conducted the study, Shi Liu analyzed the results, Cui Xu draw the figures and tables, Ying Gong designed the study.

\section{Acknowledgments}

All the enrolled patients contributed this study.

\section{Reference}

1. Choi, Y.-J. and H.J. Ra, Patient Satisfaction after Total Knee Arthroplasty. Knee surgery \& related research, 2016. 28(1): p. 1-15.

2. García-López, J., M. Polanco-García, and A. Montes, Factors Associated With the Risk of Developing Moderate to Severe Acute Postoperative Pain After Primary Total Knee Arthroplasty: Results From the PAIN OUT Registry. The Journal of Arthroplasty, 2021. 36(6): p. 1966-1973. 
3. Tetzlaff, J., Cardiovascular consequences of severe acute pain. Practical Pain Management, 2004. 4(2).

4. Miao, J., et al., An uncommon case of lower limb deep vein thrombosis with multiple etiological causes. The American journal of case reports, 2017. 18: p. 313.

5. Salleh, M.R., Life event, stress and illness. The Malaysian journal of medical sciences: MJMS, 2008. 15(4): p. 9.

6. Wasan, A.D., M. Artamonov, and S.S. Nedeljkovic, Delirium, depression, and anxiety in the treatment of cancer pain. Techniques in Regional Anesthesia and Pain Management, 2005. 9(3): p. 139-144.

7. Jia, X.-f., et al., Comparison of intrathecal and local infiltration analgesia by morphine for pain management in total knee and hip arthroplasty: a meta-analysis of randomized controlled trial. International Journal of Surgery, 2017. 40: p. 97-108.

8. Gibbs, D., T. Green, and C. Esler, The local infiltration of analgesia following total knee replacement: a review of current literature. The Journal of bone and joint surgery. British volume, 2012. 94(9): p. 1154-1159.

9. Fang, Y., et al., Local infiltration analgesia in primary total knee arthroplasty. Hong Kong Med J, 2019. 25(4): p. 279-86.

10. Mollereau, C., M. Roumy, and J.-M. Zajac, Opioid-modulating peptides: mechanisms of action. Current topics in medicinal chemistry, 2005. 5(3): p. 341-355.

11. Yuan, C.-S., Methylnaltrexone mechanisms of action and effects on opioid bowel dysfunction and other opioid adverse effects. Annals of Pharmacotherapy, 2007. 41(6): p. 984-993.

12. Ehrchen, J.M., J. Roth, and K. Barczyk-Kahlert, More than suppression: glucocorticoid action on monocytes and macrophages. Frontiers in immunology, 2019. 10: p. 2028.

13. Becker, D.E., Basic and clinical pharmacology of glucocorticosteroids. Anesthesia progress, 2013. 60(1): p. 25-32.

14. Wang, Y. and A. Zhou, A new improvement: subperiosteal cocktail application to effectively reduce pain and blood loss after total knee arthroplasty. Journal of orthopaedic surgery and research, 2020. 15(1): p. 1-6.

15. Jinlei, L., et al., Effects of intravenous tranexamic acid combined with periarticular multipoint injection of tranexamic acid cocktail on blood loss and pain after total knee arthroplasty. Chinese Journal of Tissue Engineering Research, 2021. 25(18): p. 2833.

16. Bourne, E., C. Wright, and C. Royse, A review of local anesthetic cardiotoxicity and treatment with lipid emulsion. Local and regional anesthesia, 2010. 3: p. 11-19.

17. Yu, S., et al., The development of local anesthetics and their applications beyond anesthesia. Int J Clin Exp Med, 2019. 12(12): p. 13203-13220.

18. Zhao, X. and L. Ji, Flurbiprofen axetil: Analgesic effect and adverse reaction. Pakistan journal of pharmaceutical sciences, 2018. 31. 
19. Luo, X., F. Lv, and M. Peng, Analgesic effect of different dosage of Flurbiprofen axetil in laparoscopic cholecystectomy in comparison with other analgesic drugs. Pakistan journal of pharmaceutical sciences, 2017. 30.

20. Zhang, H., et al., Absorption kinetics of flurbiprofen axetil microspheres in cerebrospinal fluid: $A$ pilot study. International journal of clinical pharmacology and therapeutics, 2017. 55(11): p. 875.

21. Tejashree, R. and B. Hemalatha, The Effect of Flurbiprofen Lozenge on Pain, Oedema, and Trismus after Impacted Third Molar Surgery: A Prospective Randomized, Double-Blinded, Placebo-Controlled Study. Sch J Dent Sci, 2021. 5: p. 160-165.

22. Lambkin-Williams, R., A. Mann, and A. Shephard, Inhibition of viral and bacterial trigger-stimulated prostaglandin E2 by a throat lozenge containing flurbiprofen: An in vitro study using a human respiratory epithelial cell line. SAGE Open Medicine, 2020. 8: p. 2050312120960568.

23. De Bodo, R., The antidiuretic action of morphine, and its mechanism. Journal of Pharmacology and Experimental Therapeutics, 1944. 82(1): p. 74-85.

24. Eidelberg, E. and A. Schwartz, Possible mechanism of action of morphine on brain. Nature, 1970. 225(5238): p. 1152-1153.

25. Rosenblum, A., et al., Opioids and the treatment of chronic pain: controversies, current status, and future directions. Experimental and clinical psychopharmacology, 2008. 16(5): p. 405-416.

26. Favié, L.M., et al., Pharmacokinetics of morphine in encephalopathic neonates treated with therapeutic hypothermia. PloS one, 2019. 14(2): p. e0211910.

27. Farsani, D.M., et al., Evaluating sedative effects of dexmedetomidine and morphine in the patients with opioid use disorder undergoing cataract surgery. American Journal of Clinical and Experimental Immunology, 2021. 10(1): p. 30.

28. Lumley, M.A. and H. Schubiner, Psychological therapy for centralized pain: an integrative assessment and treatment model. Psychosomatic medicine, 2019. 81(2): p. 114.

29. Hamm, A.O., Fear, anxiety, and their disorders from the perspective of psychophysiology. Psychophysiology, 2020. 57(2): p. e13474.

30. Barnes, P.J., Corticosteroids: the drugs to beat. European journal of pharmacology, 2006. 533(1-3): p. 2-14.

31. Claman, H.N., How corticosteroids work. Journal of Allergy and Clinical Immunology, 1975. 55(3): p. 145-151.

32. Van Staa, T., et al., Use of oral corticosteroids and risk of fractures. Journal of bone and mineral research, 2000. 15(6): p. 993-1000.

\section{Tables}

Due to technical limitations, tables are only available as a download in the Supplemental Files section.

\section{Figures}




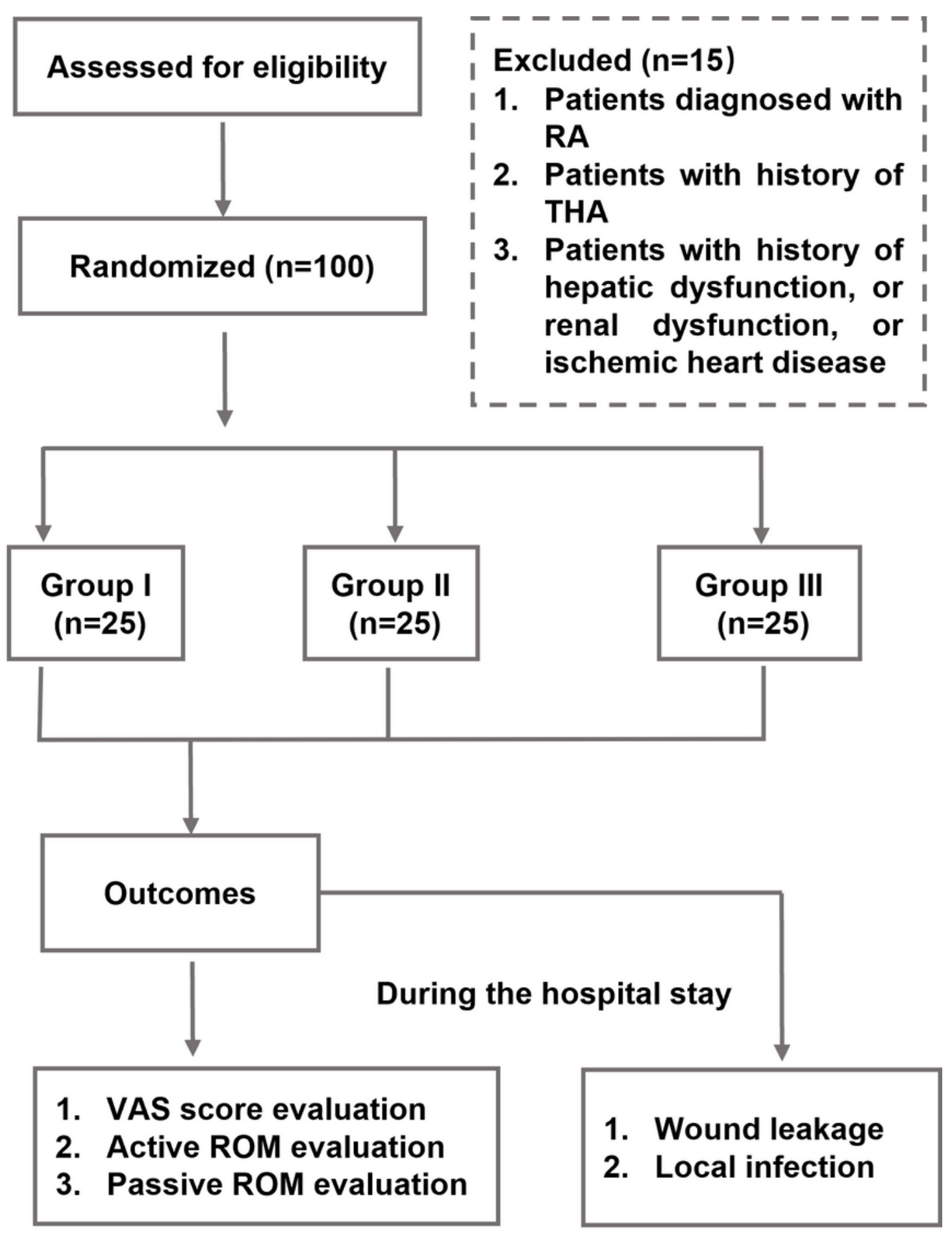

Figure 1. Flow chart of the whole study.

Figure 1

Please See image above for figure legend. 


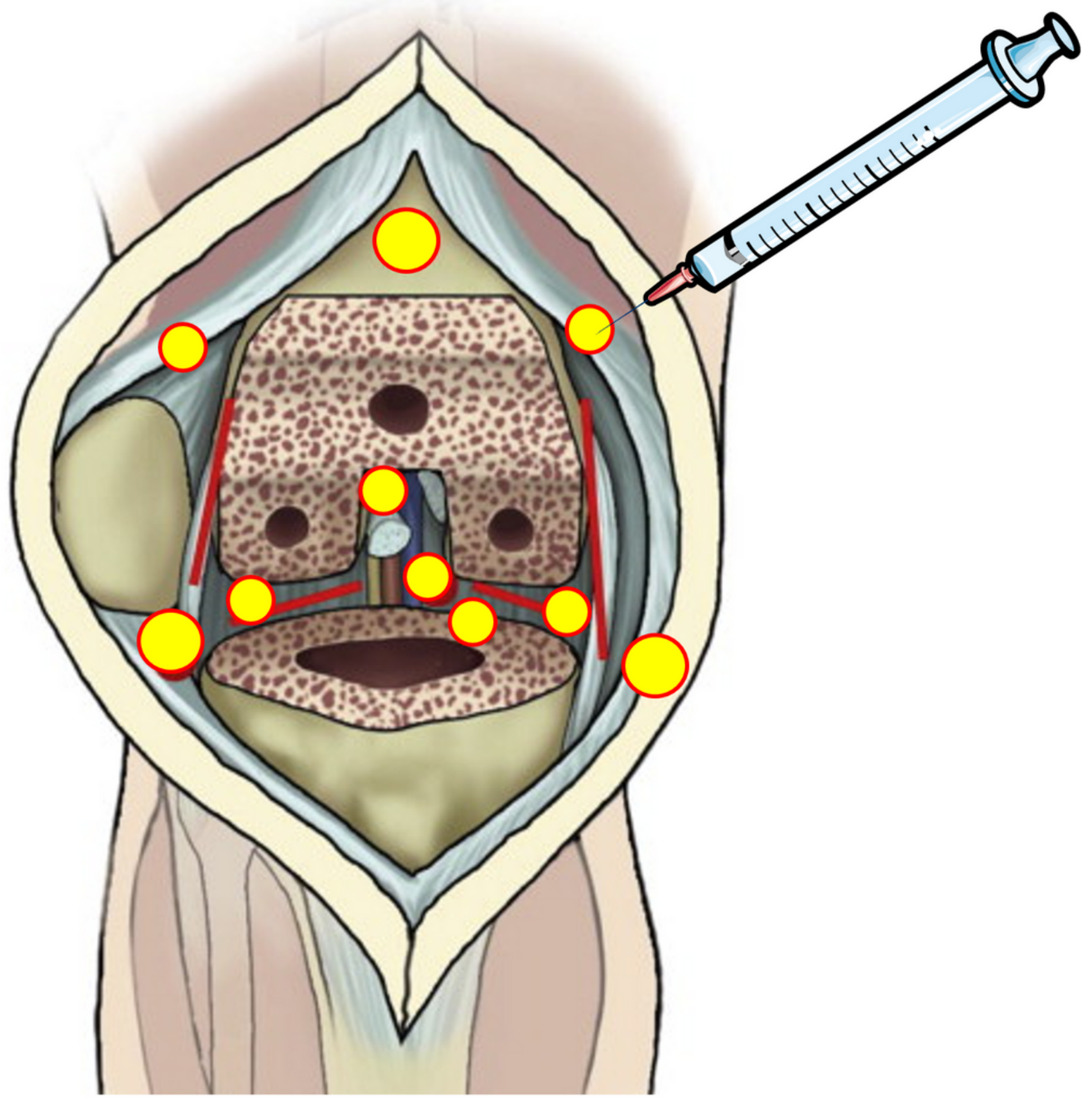

Figure 2. The local infiltration analgesia was injected at the 10 fixed locations as illustrated in the yellow round.

Figure 2

Please See image above for figure legend.

\section{Supplementary Files}

This is a list of supplementary files associated with this preprint. Click to download. 
- Table1.tif

- Table2.tif

- Table3.tif

- Table4.tif

- Table5.tif

- Table6.tif 